\title{
Kinetic Theory of Transient Condensation and Evaporation at a Plane Surface
}

\author{
P. N. Shankar* and Frank E. Marble \\ Daniel and Florence Guggenheim Jet Propulsion Center \\ California Institute of Technology, Pasadena, California 91109 \\ (Received 29 April 1970; final manuscript received 22 September 1970)
}

\begin{abstract}
The phenomenon of transient condensation onto, or evaporation from, a liquid sheet in contact with its pure vapor is treated from a kinetic theory viewpoint. The Maxwell moment method is used to formulate the detailed transient problem. A steady surface mass flux rate exists for times large in comparison with the collision time, that is, in the continuum regime, and explicit formulas are given for this limit. The complete gasdynamic field, however, is nonsteady for all times. The calculations are carried out utilizing four moments, and the effects of incorporating additional moments are negligible. Finally, the analysis is extended to incorporate imperfect mass and temperature accommodation. Examination of the transient solution and a matched asymptotic "quasisteady" solution shows that the gasdynamic field consists of a diffusion process near the liquid surface coupled through an expansion or compression wave to the constant far field state.
\end{abstract}

\section{INTRODUCTION}

The mechanism and rate of transfer of vapor to and from a liquid surface differs, depending upon whether the medium is a chemically pure substance or contains an inert diluent gas. In the latter case, only a rather small mass fraction of the diluent gas is required to make the diffusion mechanism rate controlling. Then, the vapor molecules condensing on the liquid surface must diffuse through the inert gaseous component and the rate is low in comparison with molecular velocities. For a pure substance, however, the flux rates of condensation can take place at nearly molecular velocities.

In treating this vapor transfer process between two phases of a pure substance, we have the classical formula of $\mathrm{Hertz}^{1}$ and Knudsen ${ }^{2}$ which is valid strictly in the free-molecule regime. It is assumed (see Fig. 1) that molecular flux from the liquid at temperature $T_{L}$ may be computed from the appropriate Maxwellian distribution and the number density corresponding to the saturation pressure $p_{L}$. Similarly, the flux of molecules to the surface from the surrounding vapor at ambient conditions $p_{0}, T_{0}$ is computed from a Maxwellian distribution at the vapor state $p_{0}, T_{0}$. Now, since the Maxwellian mass-flux rate ${ }^{1}$ is $p /(2 \pi R T)^{1 / 2}$, the net condensation rate per unit area, when there are no reflected molecules, is

$$
\begin{aligned}
\frac{p_{0}}{\left(2 \pi R T_{0}\right)^{1 / 2}}-\frac{p_{L}}{\left(2 \pi R T_{L}\right)^{1 / 2}} \approx \frac{1}{2 \pi} \rho_{0}\left(2 \pi R T_{0}\right)^{1 / 2} \\
\cdot\left(\frac{\left(p_{0}-p_{L}\right)}{p_{0}}-\frac{1}{2} \frac{\left(T_{0}-T_{L}\right)}{T_{0}}\right),
\end{aligned}
$$

the approximation on the right holding for small differences between vapor and saturation states.
PURE VAPOR

$$
P_{0}, T_{0}
$$

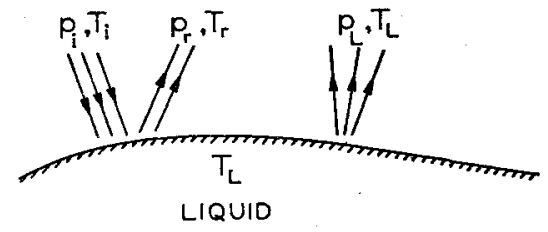

FIG. 1. The surface of a liquid in contact with its pure vapor.

Because our problem has no characteristic physical dimension, the differentiation between free molecule and continuum regimes is one of time elapsed after initiation of the process. For times small in comparison with the collision time, the transfer process is confined to the immediate neighborhood of the surface and consequently lies in the free molecule regime. For times large in comparison with the collision time, the process has spread to distances from the surface large in comparison with the mean free path and consequently lies in the continuum regime. Among obvious deductions that follow is that any steady or quasisteady surface mass flux, if such exists, occurs only after many collision times and hence only in the continuum regime. In fact, it will transpire that although a steady-state vaporization rate is achieved, the gasdynamic field is essentially nonsteady. As a consequence, it is necessary to treat the transient problem, at least in the asymptotic sense. The artificial steady problem leads either to an incorrect result, an incorrect gasdynamic field, or both. 


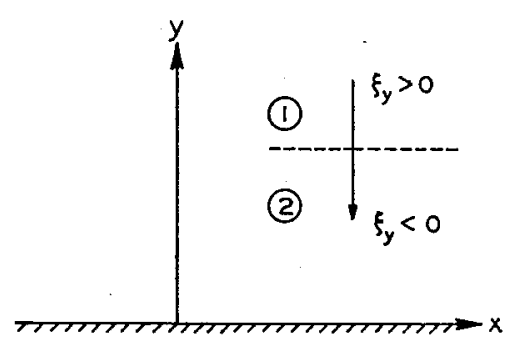

Fig. 2. Configuration for one-dimensional problem.

Consider the infinite liquid surface in contact with its pure vapor. Initially, the liquid is in equilibrium with its vapor; at $t=0$, the liquid or the vapor state is discontinuously changed. Let the vapor molecules obey an inverse fifth-power law of repulsion and satisfy the equation of state $p=\rho R T$. This description is adequate for a monatomic gas and for polyatomic gases at moderate temperatures away from the critical point. For the present, take the accommodation coefficient as unity, a restriction that will be relaxed subsequently. Assume further that the molecules are emitted from the liquid with a Maxwellian distribution corresponding to the liquid temperature and with zero mean velocity.

The kinetic theory treatment of the vapor phase involves the solution of the Maxwell-Boltzmann equation $^{3}$ for the one-particle distribution function $f$ in the absence of external forces. Because we are mainly interested in the mean flow quantities rather than the distribution function itself, the basis for this analysis will be taken as the Maxwell integral equation of transfer ${ }^{4}$

$$
\begin{aligned}
\frac{\partial}{\partial t} \int f Q\left(\xi_{i}\right) d \xi+ & \nabla_{\mathbf{r}} \cdot \int f \xi Q\left(\xi_{i}\right) d \xi \\
& =\int Q\left(\xi_{i}\right)\left(\frac{\partial f}{\partial t}\right)_{\text {coll }} d \xi=\Delta Q
\end{aligned}
$$

where $\xi$ is the vector in molecular velocity space. This moment equation is obtained by multiplying Boltzmann's equation by a function $Q\left(\xi_{i}\right)$ of the components of the molecular velocity and integrating over the velocity space. The procedure is then to express $f$ by a number of unknown functions of space and time, and write an appropriate number of moment equations to determine these functions. In this way, the Boltzmann equation is satisfied in some average sense. ${ }^{3}$

\section{FOUR MOMENT FORMULATION AND SOLU- TION}

At time $t=0$ consider the vapor at uniform temperature $T_{0}$ and pressure $p_{0}$, in contact with the liquid uniformly at temperature $T_{L} \neq T_{0}$. Evapora- tion or condensation will take place as $T_{L} \gtrless T_{0}$. We wish to calculate the steady mass flux flowing from or to the liquid surface for large time.

To formulate this problem utilizing the moment technique, choose the distribution function $f(y, \xi, t)$ of the vapor molecules ${ }^{5}$ to be given by two Maxwellian streams, Fig. 2,

$$
f(y, \xi, t)=\left\{\begin{aligned}
f_{1}= & \frac{n_{1}(y, t)}{\left[2 \pi R T_{1}(y, t)\right]^{3 / 2}} \\
& \cdot \exp \left(-\frac{\xi^{2}}{2 R T_{1}(y, t)}\right) \text { for } \xi_{y}>0 \\
f_{2}= & \frac{n_{2}(y, t)}{\left[2 \pi R T_{2}(y, t)\right]^{3 / 2}} \\
& \cdot \exp \left(-\frac{\xi^{2}}{2 R T_{2}(y, t)}\right) \text { for } \xi_{y}<0
\end{aligned}\right.
$$

where $n_{1}, T_{1}, n_{2}$, and $T_{2}$ are four unknown functions of $y$ and $t$, to be determined from the moment equations and the boundary conditions. The density $\rho$, mass velocity $\rho v$, pressure $p$, and the $y$ component of heat flux $q_{y}$, may be calculated as moments of the distribution function by choosing $Q=m, m \xi_{y}$, $\frac{1}{2} m \xi^{2}$, and $\frac{1}{2} m \xi^{2} \xi_{y}$, respectively, where $m$ is the molecular mass. Using the distribution function (2), the mean flow quantities can be evaluated in terms of $n_{1}, T_{1}, n_{2}$, and $T_{2}$. For example,

$$
\begin{aligned}
\rho v(y, t)= & \int_{-\infty}^{\infty} \int_{0}^{\infty} \int_{-\infty}^{\infty} m \xi_{y} f_{1} d \xi_{x} d \xi_{y} d \xi_{z} \\
& +\int_{-\infty}^{\infty} \int_{-\infty}^{0} \int_{-\infty}^{\infty} m \xi_{y} f_{2} d \xi_{x} d \xi_{y} d \xi_{z} \\
= & \left(\frac{R}{2 \pi}\right)^{1 / 2} m\left\{n_{1}(y, t)\left[T_{1}(y, t)\right]^{1 / 2}\right. \\
& \left.-n_{2}(y, t)\left[T_{2}(y, t)\right]^{1 / 2}\right\} .
\end{aligned}
$$

The corresponding moment equations follow in turn by setting $Q$ equal to $m, m \xi_{y}, \frac{1}{2} m \xi^{2}$, and $\frac{1}{2} m \xi_{y} \xi^{2}$ in Eq. (2):

$$
\begin{gathered}
\frac{\partial \rho}{\partial t}+\frac{\partial}{\partial y}(\rho v)=0 \\
\rho \frac{\partial v}{\partial t}+\rho v \frac{\partial v}{\partial y}-\frac{\partial P_{y y}}{\partial y}=0 \\
\frac{\partial p}{\partial t}+\frac{\partial}{\partial y}(p v)+\frac{2}{3} \frac{\partial q_{y}}{\partial y}-\frac{2}{3} P_{y y} \frac{\partial v}{\partial y}=0 \\
\frac{1}{2} \frac{\partial}{\partial t} \int m \xi_{\nu} \xi^{2} f d \xi+-\frac{1}{2} \frac{\partial}{\partial y} \int m \xi_{y}^{2} \xi^{2} f d \xi \\
=\frac{p}{\mu}\left(-\frac{2}{3} q_{\nu}+v p_{\nu \nu}\right)
\end{gathered}
$$


In evaluating these, we have accounted for the absence of external forces and the fact that $m$, $m \xi_{y}$, and $\frac{1}{2} m \xi^{2}$ are collisional invariants. The first three are the familiar continuum conservation equations. The stress $P_{y y}$ is the appropriate component of the stress tensor, $p_{y y}=P_{y y}+p$, and $\mu$ is the viscosity. The initial and boundary conditions are

$$
\begin{array}{r}
\text { for } y \geq 0, \quad t \leq 0: \\
T=T_{0}, \quad p=p_{0}, \quad v=0,
\end{array}
$$

for $y \rightarrow \infty, \quad$ all $t$ :

$$
T \rightarrow T_{0}, \quad p \rightarrow p_{0}, \quad v \rightarrow 0,
$$

for $y=0, \quad t>0$ :

$$
T_{1}(y, t)=T_{L}, \quad n_{1}(y, t)=n_{L}
$$

Condition (5b) states that all flow quantities approach the ambient state far from the liquid surface. Condition (5c) implies diffuse re-emission from the liquid at a known temperature and number density or pressure.

When $T_{0}$ and $p_{0}$ differ only slightly from $T_{L}$ and the saturation pressure corresponding to $T_{L}$, a perturbation solution may be considered:

$$
\begin{aligned}
& n_{1}(y, t)=n_{0}\left[1+\lambda N_{1}(y, t)+O\left(\lambda^{2}\right)\right], \\
& n_{2}(y, t)=n_{0}\left[1+\lambda N_{2}(y, t)+O\left(\lambda^{2}\right)\right], \\
& T_{1}(y, t)=T_{0}\left[1+\lambda t_{1}(y, t)+O\left(\lambda^{2}\right)\right], \\
& T_{2}(y, t)=T_{0}\left[1+\lambda t_{2}(y, t)+O\left(\lambda^{2}\right)\right],
\end{aligned}
$$

where $\lambda$ is a perturbation parameter that may be associated with the displacement of pressure and temperature from equilibrium $T_{0}, p_{0}$. Thus, the mean flow quantities can then be evaluated as perturbation expansions

$$
\begin{aligned}
\rho & =T_{0}\left(1+\lambda \frac{1}{2} N_{+}+\cdots\right), \\
\rho v & =\frac{\rho_{0} \beta}{2 \pi}\left[\lambda\left(N_{-}+\frac{1}{2} t_{-}\right)+\cdots\right], \\
p & =\rho R T=p_{0}\left[1+\lambda\left(\frac{1}{2} N_{+}+\frac{1}{2} t_{+}\right)+\cdots\right], \\
q_{v} & =\frac{p_{0} \beta}{2 \pi}\left[\lambda\left(7 t_{-}-2 N_{-}\right)+\cdots\right],
\end{aligned}
$$

where we denote $N_{ \pm}=N_{1} \pm N_{2}, t_{ \pm}=t_{1} \pm t_{2}$. and $\beta=\left(2 \pi R T_{0}\right)^{1 / 2}$.
The first-order perturbation problem consists in finding functions $N_{+}, N_{-}, t_{+}, t_{-}$, which satisfy the four moment equations (4) and the boundary and initial conditions (5). Introduce the dimensionless independent variables

$$
\mathrm{t}=\frac{t}{\tau_{f}} ; \quad \mathrm{y}=\frac{y}{\beta \tau_{f}},
$$

where $\tau_{f}=(\pi / 4)\left(\mu_{0} / p_{0}\right)$ is the characteristic collision time. Then, the moment equations (4) are

$$
\begin{aligned}
\frac{\partial}{\partial \mathrm{t}}\left(N_{+}\right)+\frac{1}{2 \pi} \frac{\partial}{\partial \mathrm{y}}\left(2 N_{-}+t_{-}\right) & =0, \\
\frac{\partial}{\partial \mathrm{t}}\left(2 N_{-}+t_{-}\right)+\frac{\partial}{\partial \mathrm{y}}\left(N_{+}+t_{+}\right) & =0,
\end{aligned}
$$

$$
\frac{\partial}{\partial \mathrm{t}}\left(3 N_{+}+3 t_{+}\right)+\frac{1}{\pi} \frac{\partial}{\partial \mathrm{y}}\left(4 N_{-}+6 t_{-}\right)=0,
$$

$\frac{\partial}{\partial \mathrm{t}}\left(4 N_{-}+6 t_{-}\right)+\frac{1}{2} \frac{\partial}{\partial \mathrm{y}}\left(5 N_{+}+10 t_{+}\right)$

$$
+\frac{\pi}{12}\left(7 t_{-}-2 N_{-}\right)=0
$$

and the boundary and initial conditions become

$$
\left.\begin{array}{c}
t_{+}(0, t)+t_{-}(0, t)=\frac{2 \Delta T}{\lambda T_{0}}=2 t_{I L} \\
N_{+}(0, t)+N_{-}(0, t)=\frac{2 \Delta n}{\lambda n_{0}}=2 N_{I L} \\
N_{+}(\infty, t)=0, \quad t_{+}(\infty, t)=0 \\
t_{+}(y, t)=0, \quad t_{-}(y, t)=0 \\
N_{+}(y, t)=0, \quad N_{-}(y, t)=0
\end{array}\right\} \quad t>0,
$$

The system of linear, first-order partial differential equations (9) subject to conditions (10) is solved conveniently by the method of Laplace transforms; the details of the calculation are given in Ref. 6 . Because our interest lies in the solution for large time $\left(t \gg \tau_{f}\right)$, we investigate the transform for small values of $s$. The origin in the transform $s$ plane contains the singularity with the largest real part. Therefore, for a fixed $y$, expand the transformed variables in ascending power series of $s$. The leading terms in the transforms for $p$ and $v$ are

$$
\begin{aligned}
\tilde{p}(s) & =p_{0}\left(\frac{1}{s}+\lambda\left(\frac{1}{2} \tilde{N}_{+}+\frac{1}{2} \tilde{t}_{+}\right)+O\left(\lambda^{2}\right)\right)=p_{0}\left(\frac{1}{s}+\lambda\left[\frac{5}{6} A_{2} \exp \left(w_{2} y\right)+\cdots\right]+\cdots\right), \\
\tilde{v}(s) & =\frac{\lambda \beta}{2 \pi}\left(\tilde{N}_{-}+\frac{1}{2} \tilde{t}_{-}\right)+O\left(\lambda^{2}\right) \\
& =\frac{\lambda \beta}{2 \pi}\left\{\left[(3)^{1 / 2}(s)^{1 / 2}+\cdots\right] A_{1} \exp \left(w_{1} \mathrm{y}\right)+\left[\pi\left(\frac{5}{6 \pi}\right)^{1 / 2}+\cdots\right] A_{2} \exp \left(w_{2} \mathrm{y}\right)\right\}+\cdots,
\end{aligned}
$$


where

$$
\begin{gathered}
w_{1}(s)=-\pi /(3)^{1 / 2}(s)^{1 / 2}+o(s)^{1 / 2}, w_{2}(s)=-\left(\frac{6 \pi}{5}\right)^{1 / 2} s+o(s), \\
A_{1}(s)=\frac{2}{s} \frac{\left\{\left[1+(7 \pi / 8)(5 / 6 \pi)^{1 / 2}\right] t_{I L}-\left[\frac{2}{3}+(\pi / 4)(5 / 6 \pi)^{1 / 2}\right] N_{I L}\right\}}{\left[-\frac{5}{3}-(9 \pi / 8)(5 / 6 \pi)^{1 / 2}\right]}, \\
A_{2}(s)=\frac{2}{s} \frac{\left(t_{I L}+N_{I L}\right)}{\left[\frac{5}{3}+(9 \pi / 8)(5 / 6 \pi)^{1 / 2}\right]}+\cdots .
\end{gathered}
$$

Now, $\exp \left[-\pi /(3)^{1 / 2}(s)^{1 / 2} y\right]$ leads to a solution that is diffusive in character, while exp $\left[-(6 \pi / 5)^{1 / 2} s y\right]$ gives a shift of the form $\left(1 / \tau_{f}\right)\left(t-y / a_{0}\right)$ where $a_{0}=\left(\frac{5}{3} R T_{0}\right)^{1 / 2}$ is the isentropic speed of sound. Thus, for large time, the solutions are

$$
\begin{aligned}
& p(\mathrm{y}, \mathrm{t})=\left\{\begin{array}{lll}
p_{0} & \text { for } & 0<\mathrm{t}<(6 \pi / 5)^{1 / 2} \mathrm{y} \\
p_{0}\left[1+\delta_{1}\right] & \text { for } & \mathrm{t}>(6 \pi / 5)^{1 / 2} \mathrm{y}
\end{array}\right. \\
& v(\mathrm{y}, \mathrm{t})= \begin{cases}\frac{\delta_{2}}{(\pi \mathrm{t})^{1 / 2} \exp \left(-\frac{\pi^{2} \mathrm{y}^{2}}{12 \mathrm{t}}\right)} & 0<\mathrm{t}<\left(\frac{6 \pi}{5}\right)^{1 / 2} \mathrm{y} \\
\frac{\delta_{2}}{(\pi \mathrm{t})^{1 / 2}} \exp \left(-\frac{\pi^{2} \mathrm{y}^{2}}{12 \mathrm{t}}\right)+\left(\frac{3}{10 \pi}\right)^{1 / 2} \beta \delta_{1} & \mathrm{t}>\left(\frac{6 \pi}{5}\right)^{1 / 2} \mathrm{y}\end{cases}
\end{aligned}
$$

where

$$
\begin{gathered}
\delta_{1}=\frac{1}{2} \frac{5}{3} \frac{2}{\left[\frac{5}{3}+(9 \pi / 8)(5 / 6 \pi)^{1 / 2}\right]} \frac{\Delta p}{p_{0}} \\
\delta_{2}=\frac{\beta}{2 \pi} \frac{(3)^{1 / 2}}{2} \frac{2\left\{\left[\frac{2}{3}+(\pi / 4)(5 / 6 \pi)^{1 / 2}\right]\left(\Delta n / n_{0}\right)-\left[1+(7 \pi / 8)(5 / 6 \pi)^{1 / 2}\right]\left(\Delta T / T_{0}\right)\right\}}{\left[\frac{5}{3}+(9 \pi / 8)(5 / 6 \pi)^{1 / 2}\right]}
\end{gathered}
$$

The large time solution for the velocity is composed of two parts: a diffusive part and a wave part. The diffusive part vanishes rapidly away from the plate, leaving a purely inviscid solution. The pure wave travels at the isentropic sound speed, $a_{0}=$ $\left(\frac{5}{3} R T_{0}\right)^{1 / 2}$, and remains undamped. From (13) it is seen that the pressure is composed only of the wavelike part. For $t \rightarrow \infty$, the evaporation-condensation rate at the liquid surface is given by

$$
\begin{aligned}
\dot{m}(y=0) & =\rho v(y=0) \\
& =\rho_{0}\left(2 \pi R T_{0}\right)^{1 / 2} \frac{(5 / 6 \pi)^{1 / 2}}{\left[\frac{5}{3}+(9 \pi / 8)(5 / 6 \pi)^{1 / 2}\right]} \frac{\Delta p}{p_{0}} \\
& =0.148 \rho_{0}\left(2 \pi R T_{0}\right)^{1 / 2} \frac{\Delta p}{p_{0}}=0.287 \rho_{0} a_{0} \frac{\Delta p}{p_{0}}
\end{aligned}
$$

so that the vaporization rate achieves a steady value even though the nonsteady acoustic field is always present. In particular it is to be noted that the evaporation or condensation rate depends only upon the difference between ambient vapor pressure and the saturation pressure corresponding to the liquid temperature. Thus, the continuum result, which is what we obtain for $t \gg \tau_{f}$, represents a significant departure from the Hertz-Knudsen formula.

\section{ONE-DIMENSIONAL QUASISTEADY SOLU- TION}

The asymptotic analysis demonstrates conclusively that, in the continuum regime, the condensation mechanism separates into two parts. One is confined to the neighborhood of the surface; the other consists of an expansion or compression wave propagating away from the surface and joining the local solution to the far field. On the basis of physical conjecture, one of the authors, ${ }^{7}$ applied this mechanism to estimate steady-state condensation from a continuum. The Hertz-Knudsen exchange was retained between the surface and an unknown intermediate state related to the continuum through conservation relations. The continuum was then coupled to the remote stationary vapor state through an isentropic expansion fan. This calculation led to a form identical to Eq. (16) but with numerical constant 0.145 instead of 0.148 . The favorable result of this calculation and the observed structure of the asymptotic solution suggest the possibility of a simple quasisteady solution to the four moment 
problem. The aim is to solve the steady-state moment equations with known boundary conditions at the liquid surface and with uniform $\left(p=p_{c}\right.$, $T=T_{\mathrm{c}}$ ) but unknown conditions far from the surface. This unknown state $\left(p_{c}, T_{c}\right)$ would then be coupled to the uniform state at infinity $\left(p_{0}, T_{0}\right)$ through a wave.

Deleting time dependent terms from Eqs. (9), integrating and rearranging, the four moment equations are

$$
\begin{gathered}
2 N_{-}+t_{-}=B_{1}, \\
N_{+}+t_{+}=B_{2}, \\
-N_{-}+\frac{7}{2} t_{-}=B_{3}, \\
5 N_{+}+10 t_{+}=B_{4}-\frac{4}{3} \frac{p_{0}}{\beta \mu_{0}} B_{3} y .
\end{gathered}
$$

Similarly, the corresponding linearized boundary conditions are: At $y=0$

$t_{1}^{(1)}=\frac{T_{I L}-T_{0}}{\lambda T_{0}}=\frac{\Delta T}{\lambda T_{0}}, \quad N_{1}^{(1)}=\frac{n_{I L}-n_{0}}{\lambda n_{0}}=\frac{\Delta n}{\lambda n_{0}} ;$

for $y \rightarrow \infty$ :

$$
p \rightarrow p_{c}, \quad v \rightarrow v_{c},
$$

where $p_{c}$ and $v_{c}$ are the uniform pressure and velocity behind the wave. To insure finiteness, set $B_{3}=0$, implying no heat flux to first order. Then three constants remain to satisfy four conditions (18) and the problem seems to be overspecified. This difficulty is identical to that encountered in the perturbation solution to the one-dimensional heat convection with finite mass flux. Guided by the latter problem, we seek a matched asymptotic expansion to overcome the difficutly.

Define an outer variable $\eta$ by

$$
\eta=\lambda y, \quad \frac{d}{d y}=\lambda \frac{d}{d \eta},
$$

and outer expansions of the form

$$
\begin{aligned}
& n_{1}(\eta)=n_{0}\left[1+\lambda N_{1}^{(1)}(\eta)+\lambda^{2} N_{1}^{(2)}(\eta)+\cdots\right], \\
& T_{1}(\eta)=T_{0}\left[1+\lambda t_{1}^{(1)}(\eta)+\lambda^{2} t_{1}^{(2)}(\eta)+\cdots\right] .
\end{aligned}
$$

Now, if the time independent moment equations are written in terms of $\eta$, linearized, and integrated, it is easy to show that the outer solution is given by

$$
\begin{aligned}
t_{+}(\eta) & =\frac{2}{5} B_{2}+C \exp \left(\frac{4 \pi}{3} \frac{p_{0} v^{(1)}}{\mu_{0} \beta} \eta\right), \\
N_{+}(\eta) & =\frac{3}{5} B_{2}-C \exp \left(\frac{4 \pi}{3} \frac{p_{0} v^{(1)}}{\mu_{0} \beta} \eta\right), \\
t_{-}(\eta) & =\frac{1}{8} B_{1}, N_{-}(\eta)=\frac{7}{5} B_{1} .
\end{aligned}
$$

In writing (21), a partial matching with the inner solution (17) has been used. Now to the outer solution we apply the boundary condition (18), i.e., for $\eta \rightarrow \infty: p \rightarrow p_{c}, v \rightarrow v_{c}$. This leads to the values

$$
\begin{aligned}
& B_{2}=\frac{2\left(p_{c}-p_{0}\right)}{\lambda p_{0}}, \\
& B_{1}=\frac{4 \pi v_{c}}{\lambda \beta} .
\end{aligned}
$$

It is important to note that Eq. (21) requires that $v^{(1)}$ be negative in order that the temperature field be finite. In this formulation, therefore, the steadystate problem is properly posed only for condensation. Physically, it seems reasonable that the velocity must oppose the temperature gradient in order to maintain finite temperature infinitely far away.

Proceeding with the steady solution, it is clear that the outer expansion (21) can be matched to the inner expansion (17) with a proper choice of the constant $C$. Application of conditions (18) to the inner solution then yields

$$
v_{c}=\beta \frac{8}{9 \pi} \frac{\left(p_{L}-p_{c}\right)}{p_{0}},
$$

where $v_{c}$ and $p_{c}$ are the uniform velocity and pressure behind the wave.

One may now reason that the transient condensation problem has an analogy in the "piston-tube" problem of gas dynamics where the "piston" moves away from the gas with the velocity of condensation. The pressure ratio across the expansion fan would then be

$$
p_{c}=\left(1+\frac{\gamma-1}{2} \frac{v_{c}}{a_{0}}\right)^{2 \gamma /(\gamma-1)} .
$$

Linearizing and setting $\gamma=\frac{5}{3}$ for a monatomic gas:

$$
p_{c} \approx p_{0}\left[1+\left(\frac{10 \pi}{3}\right)^{1 / 2} \frac{v_{c}}{\beta}\right] \text {. }
$$

By combining the results (23) and (25), we obtain the first-order condensation mass flux by the quasisteady-state approach:

$$
\begin{aligned}
\dot{m}(y=0) & =\rho v \\
& =\rho_{0} \beta \frac{8 / 9 \pi}{\left[1+(8 / 9 \pi)(10 \pi / 3)^{1 / 2}\right]} \frac{\Delta p}{p_{0}} \\
& =\rho_{0} \beta \frac{(5 / 6 \pi)^{1 / 2}}{\left[\frac{5}{3}+(9 \pi / 8)(5 / 6 \pi)^{1 / 2}\right]} \frac{\Delta p}{p_{0}}
\end{aligned}
$$

This is identical to the asymptotic result (16) obtained from the transient calculation. One may therefore have confidence in the quasisteady model for condensation, although this simple picture is not applicable for evaporation.

Finally, we note that although the expansion fan 
is essential in the one-dimensional calculation, it will become infinitesimally weak in the case of a spherical droplet. This is a consequence of the attenuation as $r^{-1}$ of inviscid waves in spherical geometry. We may anticipate a simpler coupling with the far field for the spherical geometry.

\section{THE EFFECT OF HIGHER MOMENTS}

The foregoing calculations have used four moment equations to determine the four unknown functions employed in representing approximately the distribution function. One's confidence in such an approximate integral method is greatly increased if it can be shown that the physical results are little changed by the use of higher moments. To demonstrate the relative insensitivity of the present solution to the details, we shall examine a six-moment solution for the same problem. Let the distribution function (Fig. 2) be represented by the two-stream Maxwellian

$$
f(y, \xi, t)=\left\{\begin{array}{c}
f_{1}=\frac{n_{1}(y, t)}{\left[2 \pi R T_{1}(y, t)\right]^{3 / 2}} \exp \left(-\frac{\xi_{x}^{2}+\xi_{x}^{2}+\left[\xi_{y}-v_{1}(y, t)\right]^{2}}{2 R T_{1}(y, t)}\right) \\
f_{2}=\frac{n_{2}(y, t)}{\left[2 \pi R T_{2}(y, t)\right]^{3 / 2}} \exp \left(-\frac{\xi_{x}^{2}+\xi_{z}^{2}+\left[\xi_{y}-v_{2}(y, t)\right]^{2}}{2 R T_{2}(y, t)}\right)
\end{array}\right.
$$

where $n_{1,2}(y, t), T_{1,2}(y, t), v_{1,2}(y, t)$ are six undetermined functions. This is the most obvious extension of the four-function approach, since it is the $y$ velocity that we are trying to calculate.

The mean flow quantities can now be determined in terms of the six unknown functions $n_{1,2}, T_{1,2}, v_{1,2}$. The integrations are now complicated by the presence of $v_{1}$ and $v_{2}$ in the exponentials, but since we shall solve only the linearized problem it is advantageous to evaluate the mean flow quantities after linearizing $n_{1}, n_{2}$, etc., in the manner of Eqs. (6). The velocities

$$
v_{1,2}=\beta\left[\lambda \nu_{1,2}(y, t)+\cdots\right]
$$

have no zeroth-order parts since they vanish at equilibrium.

Then, for example, the density is given to first order by

$$
\begin{aligned}
\rho(y, t)= & \int m f d \xi=\int_{-\infty}^{\infty} \int_{0}^{\infty} \int_{-\infty}^{\infty} m f_{1} d \xi_{x} d \xi_{y} d \xi_{z} \\
& +\int_{-\infty}^{\infty} \int_{-\infty}^{0} \int_{-\infty}^{\infty} m f_{2} d \xi_{x} d \xi_{y} d \xi_{z} \\
= & \rho_{0}\left[1+\lambda\left(\frac{1}{2} N_{+}+\nu_{-}\right)+O\left(\lambda^{2}\right)\right],
\end{aligned}
$$

where

$$
N_{+}=N_{1}+N_{2}, \quad \nu_{-}=\nu_{1}-\nu_{2} \text {, etc. }
$$

The six equations to be used correspond to the following moments: $Q=m, m \xi_{y}, \frac{1}{2} m \xi^{2}, m \xi_{x}^{2}, \frac{1}{2} m \xi_{y} \xi^{2}$, and $m \xi_{y}^{3}$. These yield, in addition to Eqs. (4),

$$
\begin{aligned}
& \frac{\partial}{\partial t} \int m \xi_{x}^{2} f d \xi+\frac{\partial}{\partial y} \int m \xi_{y} \xi_{x}^{2} f d \xi=\frac{p}{\mu} p_{x x} \\
& \frac{\partial}{\partial t} \int m \xi_{y}^{3} f d \xi+\frac{\partial}{\partial y} \int m \xi_{\nu}^{4} f d \xi \\
& \quad=\frac{p}{\mu}\left(q_{\nu}+3 v p_{\nu y}-\frac{3}{2} \int m\left(\xi_{\nu}-v\right)^{3} f d \xi\right)
\end{aligned}
$$

The boundary conditions for the transient problem are

$$
\begin{array}{ll}
\text { for } y \rightarrow \infty: & T \rightarrow T_{0}, p \rightarrow p_{0}, v \rightarrow 0, \\
\text { at } y=0, t>0: & n_{1}=n_{L}, T_{1}=T_{L}, v_{1}=0 .
\end{array}
$$

The solution of the linearized problem by Laplace transforms is given in Ref. 6. As before, the solution is composed of a diffusive part and a wavelike part, plus a wave damped exponentially in $y$. For $t \rightarrow \infty$, the mass flux at the liquid surface is given by

$$
\dot{m}=\rho v(y=0)=\rho_{0} \beta \frac{1}{\left\{(10 \pi / 3)^{1 / 2}+\left[9 \pi+12(5 \pi / 2)^{1 / 2}\right] /\left[8+3(5 \pi / 2)^{1 / 2}\right]\right\}} \frac{\Delta p}{p_{0}}=0.143 \rho_{0} \beta \frac{\Delta p}{p_{0}} .
$$

Comparing with (16), it seems that the four-moment solution is remarkably good; the extra moments give greater detail but do not change the magnitudes of the mean quantities appreciably. The quasisteady approach again gives exactly the same result (3) for condensation. ${ }^{6}$

\section{THE EFFECT OF ACCOMMODATION CO- EFFICIENTS}

In the results we have obtained, it is assumed that molecules hitting the liquid surface are absorbed by it and re-emitted at the liquid temperature and vapor pressure. Although this assumption is 
often a reasonable one, it is possible to relax the condition somewhat. Let the mass flux accommodation coefficient $\alpha_{m}$ and the energy accommodation coefficient $\alpha_{T}$ be defined as follows:

$$
\begin{aligned}
& \alpha_{m}=\left(\dot{m}_{1}-\dot{m}_{R}\right) /\left(\dot{m}_{1}-\dot{m}_{L}\right), \\
& \alpha_{T}=\left(E_{i}-E_{R}\right) /\left(E_{i}-E_{L}\right),
\end{aligned}
$$

where $i$ refers to the incident stream, $R$ refers to the receding stream, and $L$ refers to the stream from the liquid. When the receding stream is composed only of the stream from the liquid, with the incident stream unreflected, both $\alpha_{T}$ and $\alpha_{m}$ are equal to 1 .

In the present analysis, using four moments, the incident stream is characterized by $n_{2}$ and $T_{2}$, the receding stream by $n_{1}$ and $T_{1}$, and the stream from the liquid by $n_{L}$ and $T_{L}$. In the linearized formulation

$$
\begin{gathered}
\dot{m}_{R}=\frac{\rho_{0} \beta}{2 \pi} \lambda\left(N_{1}+\frac{1}{2} t_{1}\right), \\
E_{R}=\int \frac{1}{2} m \xi_{y} \xi^{2} f d \xi=\frac{p_{0} \beta}{4 \pi} \lambda\left(N_{1}+\frac{3}{2} t_{1}\right)
\end{gathered}
$$

with similar relations for $\dot{m}_{i}, E_{i}, \dot{m}_{L}$, and $E_{L}$. Substituting these relations into (34), the boundary conditions at $y=0$ are

$$
\begin{array}{r}
t_{1}(y=0)=N_{L}\left(\alpha_{T}-\alpha_{m}\right)+t_{L}\left(\frac{3}{2} \alpha_{T}-\frac{1}{2} \alpha_{m}\right) \\
+N_{2}\left(\alpha_{m}-\alpha_{T}\right)+t_{2}\left(1+\frac{1}{2} \alpha_{m}-\frac{3}{2} \alpha_{T}\right),
\end{array}
$$

$$
\begin{aligned}
& N_{1}(y=0)=N_{L}\left(\frac{3}{2} \alpha_{m}-\frac{1}{2} \alpha_{T}\right)+t_{L}\left(\frac{3}{4} \alpha_{m}-\frac{3}{4} \alpha_{T}\right) \\
& +N_{2}\left(1-\frac{3}{2} \alpha_{m}+\frac{1}{2} \alpha_{T}\right)+t_{2}\left(\frac{3}{4} \alpha_{T}-\frac{3}{4} \alpha_{m}\right) .
\end{aligned}
$$

With these boundary conditions, the evaporating or condensing mass flux can be calculated to be

$\dot{m}=\rho_{0}\left(2 \pi R T_{0}^{\prime}\right)^{1 / 2}$

$\cdot \frac{1}{\left\{\left[\pi\left(10 \alpha_{m}-9 \alpha_{m} \alpha_{T}+8 \alpha_{T}\right) / 8 \alpha_{m} \alpha_{T}\right]+(10 \pi / 3)^{1 / 2}\right\}} \frac{\Delta p}{p_{0}}$.

When $\alpha_{m}=\alpha_{T}=1,(36)$ reduces to (16), as expected.

\section{ACKNOWLEDGMENT}

This work was performed with the financial support of the Aerospace Research Laboratories, Wright-Patterson Air Force Base, Contract No. F33615-69-C-1069.

* Present address: Department of Aerospace Engineering, University of Maryland, College Park, Maryland 20742.

1 H. Hertz, Ann. Physik 17, 177 (1882).

${ }_{2}^{2}$ M. Knudsen, Ann. Physik 47, 697 (1915).

${ }^{3} \mathrm{H}$. Grad, in Handbuch der Physik, edited by S. Flügge (Springer-Verlag, Berlin, 1958), Vol. 12, p. 205.

4 T. C. Maxwell, Collected Works (Cambridge University Press, London, 1890), Vol. II.

L. Lees, J. Soc. Indust. Appl. Math. 13, 278 (1965).

- P. N. Shankar, Ph.D. Thesis, California Institute of Technology (1968).

7 F. E. Marble, Astronaut. Acta 16, 585 (1969). 